\title{
AN IMPROVEMENT IN MAXIMUM LIKELIHOOD LOCATION ESTIMATION ALGORITHM IN SENSOR NETWORKS
}

\author{
Zeinab Abbasi ${ }^{1}$, Dr. Ahmad Farahi ${ }^{2}$, Dr. Hamid Haj Seyed Javadi ${ }^{3}$ \\ ${ }^{1}$ Technical \& engineering college, Payame noor University, Tehran, Iran \\ zabasi@gmail.com \\ ${ }^{2}$ Technical \& engineering college, Payame noor University, Tehran, Iran \\ afaraahi@pnu.ac.ir \\ ${ }^{3}$ Basis Science college, Shahed University, Tehran, Iran \\ h.s.javadi@shahed.ac.ir
}

\begin{abstract}
This paper reviews on one of the localization algorithm works based on Maximum Likelihood Estimation Method and tries to improvement the performance of this algorithm using a new method. this localization algorithm is a model-based localization algorithm which could be used to estimate location using RSS when a statistical model is available.
\end{abstract}

\section{Keywords}

Wireless sensor networks; Localization algorithm; Maximum Likelihood Estimation Method; Received signal strength.

\section{INTRODUCTION}

Recent advances in micro-electro-mechanical systems obtain the ability to design and produce sensors with low power expenditure, small size, and proper price and variety applications [1]. These sensors are incentive for appearance an idea for creation and developing networks namely the "Wireless sensor networks".

A wireless sensor network contains many sensors that Spread in an environment and gather the information from their vicinity [1]. Wireless sensor networks are tremendously being used in different environments to perform various monitoring tasks such as search, rescue, disaster relief, target tracking and a number of tasks in smart environments. May be the location of sensors are not predefined and given [10]. This feature helps to use them in hazardous or inaccessible places. These sensors after gathering the information from environment and process the data send them to control stations. Later on other parts decide and act based on received information. One of the challenging problems exists in wireless sensor network that is how to obtain location information for sensor nodes present in the network [1].

The existing localization algorithms in wireless sensor networks divide into three branches: GPS (Global Positioning System), LPS (Local Positioning System) and relative localization (sometimes called distributed or GPS-free). 
In localization with GPS method, all sensors have GPS and know their position. For outdoor applications in which device density is low, and cost is not a major concern, GPS is a viable option. But in LPS, a fraction of existing devices are reference nodes that have a priori information about their location and other nodes communicate with them and then triangulate to determine their locations based on estimated distance obtained with RSS (received signal strength), TDOA (time difference of arrival), or TOA (time-of-arrival) technologies. in this type of networks, as the fraction of GPS functionality decreases, the range of the devices must be larger, and the power drain at the GPS-functional device increases[2][6].

Another way to obtain the sensor's location in a network is to use relative localization method in wireless sensor network. in this method, for estimating the position of nodes, each node calculates the distance between itself and others. One difficulty using this method is that as more and more devices are added into the location map, the number of communication increases and the number of possible combinations will rise very rapidly to find the estimated ranges with minimum error [6].

The following steps must be doing for relative localization in a wireless sensor network:

1) First, some of nodes must be reference nodes that obtain absolute location from a way such as GPS.

2) Second, all of the nodes must be able to estimate the range between themselves and their neighbors.

3) Third, there must be an ad-hoc network protocol by which the nodes can pass along range and location estimates to other nodes.

4) Finally, there must be a 'location mapping algorithm' that estimates the locations of the nodes given the pair-wise range estimates and the known coordinate of the reference nodes [2].

This paper explores one of the existing relative localization methods that use the "Maximum Likelihood Estimation (MLE)" method for mapping algorithm in order to find the best estimated location using the estimated ranges between nodes that measures with variety ways. MLE is a preferred method of parameter estimation in statistics and is an indispensable tool for many statistical modeling techniques, in particular in non-linear modeling with non-normal data [8]. But it has some problems when using for localization.

This paper suggests a method for overcoming one of these problems that is called "local maxima". In this method based on the measured distances between sensors and the proximity value to the reference nodes, approximates sensors location, then give to the MLE algorithm for finding the final locations.

First, section 2 mentions the related works and algorithms about using MLE in localization. Next, section 3 states the relative location estimation problem. Next, section 4 describes MLE and uses it by RSS measurement to estimate the location of sensors. Section 5 will be illustrated the new proposed method for improving the MLE localization algorithm. In Section 6, the experimental results presented to validate the new method. Finally, this paper concludes with section 7.

\section{RELATED WORK}

In first, the MLE method was recommended, analyzed and vastly popularized by R. A. Fisher in 1922 (although it had been used earlier by Gauss, Laplace, Thiele, and F. Y. Edgeworth). After that it used and developed by other researchers for many application. 
For the first time, N. Patwari uses it for sensor localization problem in [2]. His presented algorithm is centralizad and based on Received Signal Strength. He completes and improves his algorithm for working the algorithm with the TOA measured data in [6].

Researchers in [4] present some improvments for the MLE algorithm and examine it in multiple actual environment and calculate the measuring parameters in each environment, the proposed method is useful for indoor personnel location management. Finally, M. Laaraiedh in [9] suggests that typical median estimator must be replaced by maximum likelihood estimator to enhance the positioning accuracy and he is proposed a new direct RSS-based estimation scheme of position.

In the every of the above mentioned researches is tried to improve the MLE algorithm. But this algorithm has some problems, yet. this paper suggests the new method for solving some difficulties.

\section{Problem Statement}

Before going into detail, it is useful to formally state the sensor location estimation problem. In the network with $N$ total sensors, the localization problem is to estimate the coordinates of $n$ of the sensors, given a priori the coordinates of $m$ of the sensors (reference nodes), where $N=n+$ $m$. In other words, for the 2-D localization problem, a total of $2 n$ unknown-location node parameters must be estimated, $\theta=\left[\theta_{\mathrm{x}}, \theta_{\mathrm{y}}\right]$, where:

$\theta_{y}=\left[y_{1}, y_{2}, \ldots, y_{n}\right], \theta_{x}=\left[x_{1}, x_{2}, \ldots, x_{n}\right]$

Also, supposed that given the m known reference coordinates as follows:

$\left[x_{n+1}, x_{n+2}, \ldots, x_{n+m}, y_{n+1}, y_{n+2}, \ldots, y_{n+m}\right]$

The location of sensor $i$ is also referred to as $z_{i}$ where $z_{i}=\left[x_{i}, y_{i}\right]$. While the 2-D case is considered in this paper, extension to $3-\mathrm{D}$ appends a third coordinate to each sensor location vector[6]. Pair-wise measurements given from sensors have the form of $\left\{X_{i, j}\right\}$ where every pair of $X_{i, j}$ is a measurement between sensor $i$ and sensor $j$. Pair-wise measurements $X_{i, j}$ could be any physical reading that indicates distance or relative position, e.g. TOA, AOA, RSS, or connectivity (whether or not two devices can communicate). Note that these pair-wise measurements could be done via various modalities e.g. RF, IR (infrared), acoustics, or a combination of these. In the case of pair-wise measurements, it is not assumed that all sensor pairs make measurements [6]. In the next part, will illustrate the MLE method and how to use of it in sensor localization problem.

\section{MaXimum Likelihood Estimation Method}

The MLE is a centralized and model-based localization algorithm which could be used to estimate location using TOA, RSS, connectivity, or AOA measurements, as long as a statistical model is available [6]. MLE estimates the position of a node by minimizing the differences between the measured distances and estimated distances [10]. MLE is a popular statistical method used for fitting a statistical model to data, and providing estimates for the model's parameters.

MLE has many optimal properties in estimation: sufficiency, consistency, efficiency and parameterization invariance [8]. 
In this method, Let the unknown parameter vector of the model be $\theta=\left(\theta_{1}, \theta_{2}, \ldots, \theta_{\mathrm{n}}\right)$, and the observed data be represented as $P=\left(P_{1}, P_{2}, \ldots, P_{n}\right)$. Suppose that the conditional probability density function of $P$ with respect to $\theta$ is given by:

$p(P \mid \theta)=p\left(P_{1}, P_{2}, \ldots, P_{n} \mid \theta\right)$.

Treating the conditional probability density function $p(P \mid \theta)$ as a function of $\theta, l(\theta):=p(P \mid \theta)$ is called the likelihood function, and $L(\theta):=\log l(\theta)$ is called the logarithmic likelihood function[4]. In general, the MLE finds the parameters which maximizes the likelihood function, or equivalently, minimizes the negative of the log-likelihood function. Thus, the $\hat{z}$ parameter obtained with this method is the estimated location for a sensor [5]. If the data is known to be described well by a particular statistical model (e.g. Gaussian or log-normal), then the localization algorithm with MLE method can be derived and implemented [6].

One reason that these estimators are used is that their variance asymptotically (as the signal-tonoise (SNR) ratio goes high) approaches the lower bound presented for this algorithm. However, there are two difficulties with this approach:

1) Local Maxima: Unless the MLE is initialized to a value close to the correct solution, it is possible that our maximization search may not find the global maximum [8].

2) Model Dependency: If measurements deviate from the assumed model (or model parameters), the results are no longer guaranteed to be optimal [6].

In the next part, the presented MLE formulas explained for $\hat{z}$ estimation in regard of measured data.

\subsection{Received Signal Strength formulation}

Received Signal Strength (RSS) is defined as the voltage measured by a receiver's received signal strength indicator (RSSI) circuit [6]. RSSI measures the power of the signal at the receiver and based on the known transmit power, the effective propagation loss can be calculated [10].

The distances measured with RSS have error and this error cause of non uniformly signal propagation in variety environments. But since RSSI has become a standard feature in most wireless devices, therefore RSS based localization techniques require no additional hardware, and they have the simplicity and low power consumption[7]. Typically, the ensemble mean received power in a real-world, obstructed channel decays proportional to $d^{-n_{p}}$, where $\mathrm{d}$ is distance between two sensors and $n_{p}$ is the 'path-loss exponent', typically between 2 and $4[6]$.

The RSS has been popularly modeled by a log-normal model, thus the random variable $P_{i, j}(\mathrm{dBm})=10 \log _{10} P_{i, j}$ is Gaussian.

$P_{i, j}(d B m) \sim \mathcal{N}\left(\bar{P}_{i, j}(d B m), \sigma_{d B}^{2}\right)$,

$\bar{P}_{i, j}(d B m)=P_{0}(d B m)-10 n_{p} \log _{10}\left(d_{i, j} / d_{0}\right)$,

Where $\bar{P}_{i, j}(\mathrm{dBm})$ is the mean power in $\mathrm{dBm}, \sigma_{\mathrm{dB}}^{2}$ is the variance of the shadowing, and $P_{0}(\mathrm{dBm})$ is the received power at a reference distance $d_{0}$ and $d_{i, j}$ is the distance between sensor $i$ and sensor $j[6][7]$.

The MLE for the RSS case is [5][6], 
$\left\{\hat{\mathrm{z}}_{\mathrm{i}}\right\}=\arg \min _{\left\{\mathrm{z}_{\mathrm{i}}\right\}} \sum_{i=1}^{N} \sum_{\substack{j \in H(i) \\ j<i}}\left(\ln \frac{\left(\delta_{i, j}^{M L E}\right)^{2}}{\left\|z_{i}-z_{j}\right\|^{2}}\right)^{2}$,

Where $\delta_{i, j}^{M L E}$ is a function of the measured received power $P_{i, j}$ and the best estimation for $\left\|z_{i}-z_{j}\right\|$ in the maximum-likelihood sense, and defined as follows,

$\delta_{i, j}^{M L E}=d_{0} 10 \frac{p_{0}-p_{i, j}}{10 n_{p}}$.

Note that $\delta_{\mathrm{i}, \mathrm{j}}^{M L E}$ has a log-normal distribution since $\log \delta_{\mathrm{i}, \mathrm{j}}^{M L E}$ has a Gaussian distribution, and that

$E\left[\delta_{i, j}^{M L E}\right]=C\left\|z_{i}-z_{j}\right\|$,

Where

$C=\exp [\gamma / 2]$,

Where

$\gamma=\left(\frac{10 n_{p}}{\sigma_{d B} \log 10}\right)^{2}$.

The parameter $C$ is a multiplicative bias factor. For typical channels, $C \approx 1.2$, adding $20 \%$ bias to the range [6].

Motivated by (7), a bias-corrected estimator (a pseudo-MLE) of distance can be defined just by dividing the MLE by $C$ [6],

$\left\{\hat{\mathrm{z}}_{\mathrm{i}}\right\}=\arg \min _{\left\{\mathrm{z}_{\mathrm{i}}\right\}} \sum_{i=1}^{N} \sum_{\substack{j \in H(i) \\ j<i}}\left(\ln \frac{\left(\delta_{i, j}^{B C}\right)^{2}}{\left\|z_{i}-z_{j}\right\|^{2}}\right)^{2}$,

Where $\delta_{i, j}^{B C}$ is the MLE of distance divided by the bias factor $C$. and is equals to [6],

$\delta_{i, j}^{B C}=\frac{d_{0}}{C} 10^{\frac{p_{0}-p_{i, j}}{10 n_{p}}}$.

Even in the bias-reduced estimator in (10), there remains residual bias.

\section{IMPROVEMENT METHOD}

This section presented a new technique for solving one of the MLE localization algorithm problems (local maxima) that mentioned in section 3.

One approach that is calculating an initial position with a simple and fast technique. This method utilizes the position of reference nodes and the distances between the reference nodes and other nodes obtained by (11) and acquire an initial guess for location of nodes. Also, this method is fully distributed and each sensor performs it independently.

This technique has four steps: 
1) First, the range of each reference node calculated to determine which sensors place in their range.

In this paper supposed that the network's environment is a $\mathrm{L} \times \mathrm{L}$ square and the range of each reference node is in the circle form, thus the radius of this circle is,

$K^{2}=L^{2}+L^{2}=2 L^{2}$, so $K=\sqrt{2 L^{2}}=L \sqrt{2}$,

$r=\left\lceil\frac{L \sqrt{2}}{2}\right\rceil$

Where $\mathrm{K}$ is the diagonal of network square.

2) Second, the weight of each sensor for each reference computes by the following formula, if the sensor is in range $r$,

$W_{i, j}=1-\frac{\delta_{i, j}^{B C}}{\mathrm{r}}$

Where $\delta_{i, j}^{B C}$ is the estimated distance between two nodes (one reference and one sensor) calculated in (11) and $\mathrm{r}$ is the range of each reference expressed in (12).

Now, each node has a vector that contains four weight values are between 0 and 1 and indicate which references are nearest it.

3) Third, each node estimates the location of itself,

$\left\{\hat{\mathrm{z}}_{\mathrm{i}}\right\}=\frac{\sum_{\mathrm{j}=1}^{\mathrm{m}}\left(\mathrm{W}_{\mathrm{i}, \mathrm{j}} \cdot \mathrm{Z}_{\mathrm{j}}\right)}{\sum_{\mathrm{j}=1}^{\mathrm{m}} \mathrm{W}_{\mathrm{i}, \mathrm{j}}}$

Where, $\mathrm{Z}_{\mathrm{j}}$ is the reference nodes coordinate

5) Finally, the estimated positions send to the central node to performing the MLE localization algorithm.

\section{Simulation Results}

In the below experiments, MLE executed on various topologies of networks in Matlab. The nodes are placed (a) randomly with a uniform distribution within a square area, (b) randomly with a C-shape distribution within a square area.

For comparison purposes, The measured $\mathrm{P}_{\mathrm{ij}}$ match the log-normal shadowing model in (4) with $\mathrm{n}_{\mathrm{p}}=2.30$ and $\sigma_{\mathrm{dB}}=3.92 \mathrm{~dB}$, using $\mathrm{d}_{0}=1 \mathrm{~m}$ [6]. The measured distance between nodes $\widehat{\mathrm{d}}_{\mathrm{ij}}$ is modeled with a noise drawn from a normally-distributed random variable with mean 0 and variance of 1 .

$\hat{d}_{i j}=\delta_{i, j}^{B C} \cdot 10^{\mathrm{X}_{\sigma}}$ Where $\mathrm{X}_{\sigma} \sim \mathcal{N}(0,1)$

Where $\delta_{i, j}^{B C}$ expressed in (11) and $\mathrm{X}_{\sigma}$ is the additive noise. The performance measure employed in the experiments is the Root Mean Square Error (RMSE) between the true location of sensor nodes and the estimated location of sensor nodes. 


\subsection{Random With A Uniform Distribution Placement}

In this experiment, 196 nodes were randomly placed in a $10 \mathrm{~m} \times 10 \mathrm{~m}$ square area. Four reference nodes are placed in the corners of square area.

Simulations were done with a 15\% distance error measurement and 30 trials for each data points. The average of RMSE for the original MLE algorithm was $2.04 \mathrm{~m}$ whereas the proposed method reduced the average RMSE to $0.63 \mathrm{~m}$. The range $\mathrm{r}$ used in this simulation was $8 \mathrm{~m}$. In another simulation with 80 nodes and four reference nodes that placed in $30 \mathrm{~m} \times 30 \mathrm{~m}$ square area the average RMSE for 20 trials decreased from $3.66 \mathrm{~m}$ in the original MLE to $2.04 \mathrm{~m}$ in modified MLE algorithm. The range $r$ was set to $22 \mathrm{~m}$.

Figure 1 shows the location estimates by the modified MLE algorithm on the before mentioned data points.

\subsection{Random With C-shape Distribution Placement}

In this experiment, 156 nodes were randomly with C-shape distribution placed in a $10 \mathrm{~m} \times 10 \mathrm{~m}$ square sensing field. Simulations were done with a $15 \%$ distance error measurement and 30 trials for each data points. The RMSE for original MLE was $2.38 \mathrm{~m}$ and it reduced to $0.72 \mathrm{~m}$ by using modified MLE. In this case the range $\mathrm{r}$ was $29 \mathrm{~m}$. Figure 2 exhibits the result of execution of the modified MLE algorithm on the nodes of this simulation.

In another test, 40 sensors in a $40 \mathrm{~m} \times 40 \mathrm{~m}$ square area the obtained RMSE for the original MLE was 4.27 and for modified MLE was 3.19. In this case the range $r$ was $29 \mathrm{~m}$. Note that the good selection for range $r$ is very necessary. For the example, in the last simulation case if $r$ was set to 28 instead of 29 , the RMSE would be 3.42 .

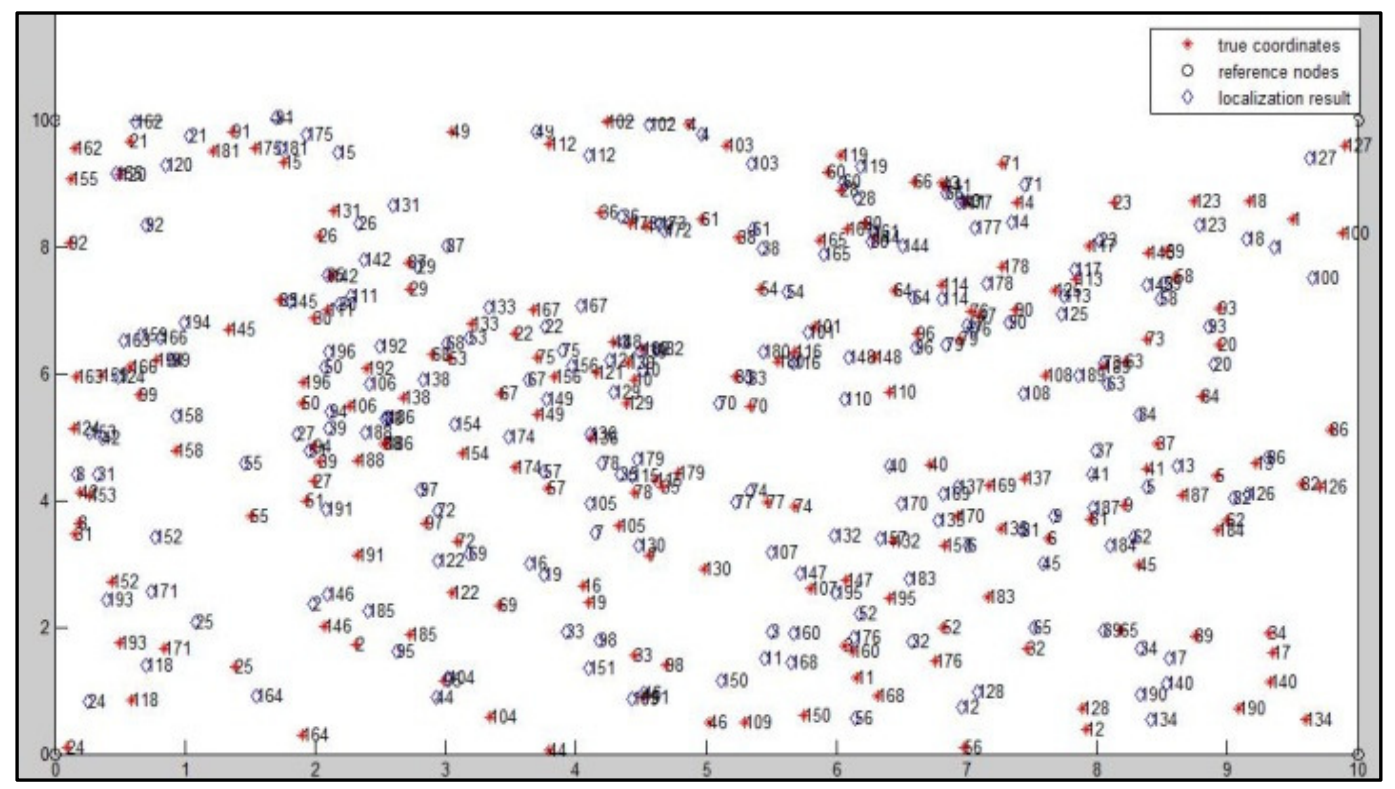

figure. 1 Position estimates for uniform random placement. 


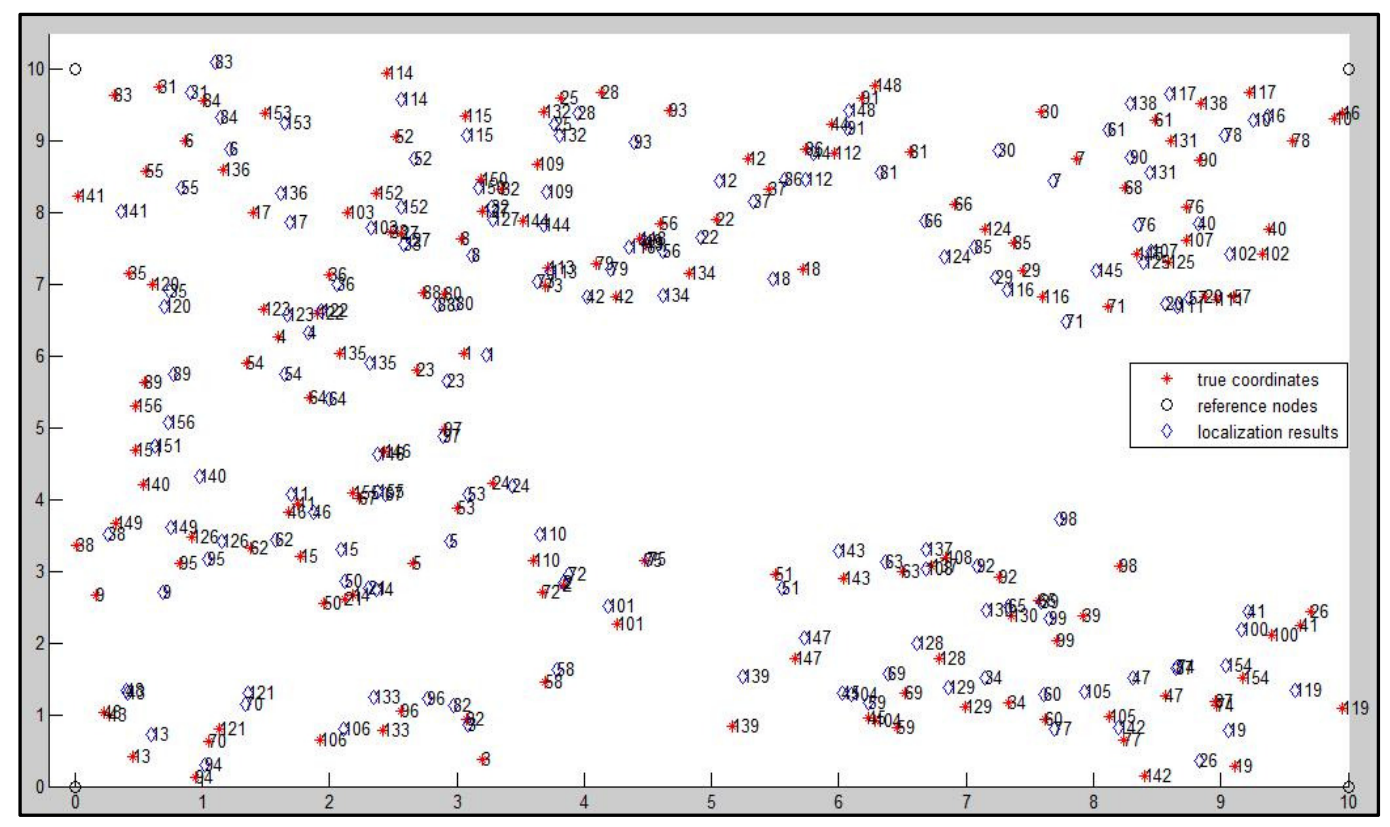

figure. 2 Position estimates for c-shape distribution random placement

\section{CONCLUSION}

In this paper, a new method proposes to improvement the performance of MLE localization algorithm. This method doesn't require to any extra hardware and it has the less complexity and results the less RMSE than the original MLE.

According to presented in [6], MLE localization algorithm takes $\mathrm{O}\left(\mathrm{LN}^{2}\right)$ time that $\mathrm{N}$ is total number of sensors and $\mathrm{L}$ is the number of iteration of MLE algorithm for achieving to the optimal solution. So the proposed method takes $\mathrm{O}\left(\mathrm{N}+\mathrm{LN}^{2}\right)$ time that is equivalent to $\mathrm{O}\left(\mathrm{LN}^{2}\right)$. However, this method takes the less time than original MLE practically, because it's a well known optimization algorithm that converges to the optimized solution faster than original MLE.

Researches in [3] shows that MLE does not take much advantage of having more reference nodes here but the proposed method maximizes the use of the reference nodes. It performs well with the less measurement error. Also similar to original MLE it performs excellently when unknown nodes are surrounded by reference nodes and the network is dense. A drawback of proposed algorithm that is it requires sufficient reference nodes (4 or more for 2-D networks, 5 or more for 3-D networks).

\section{REFERENCES}

[1] R. Poovendran, C. Wang, and S. Roy, " Secure Localization and Time Synchronization for Wireless Sensor and AdHoc Networks," America, Springer publication, 2007.

[2] N. Patwari, R. J. O'Dea, and Y. Wang, "Relative location in wireless networks," IEEE VTC, vol. 2, 2001, pp. 1149-1153.

[3] J. Desai and U. Tureli, "Evaluating performance of various localization algorithms in wireless and sensor networks," 18th Annual IEEE Symposium on Personal, Indoor and Mobile Radio Communications (Athens, September 2007). PIMRC'07, pp. 1-5, 2007. 
International Journal of Computer Science \& Engineering Survey (IJCSES) Vol.2, No.1, Feb 2011

[4] M. Takashima, and et al. , "Location estimation using received signal power and maximum likelihood estimation in wireless sensor networks," Wiley Periodicals, Electronics and Communications in Japan, vol. 90, no. 12, pp. 62 - 72, 2007.

[5] H. Cho, M. Kang, J. Kim, and H. Kim, "ZigBee Based Location Estimation in Home Networking Environments,” IEICE - Trans, vol. E90-D, no. 10, pp. 1706-1708, 2007.

[6] N. Patwari, A. O. Hero, M. Perkins, N. S. Correal, and R. J. O'Dea, "Relative location estimation in wireless sensor networks,” IEEE Trans. Signal Process, vol. 51, no. 8, pp. 2137-2148, 2003.

[7] G. Mao, B. D. O. Anderson, and B. Fidan, "Path loss exponent estimation for wireless sensor network localization," Elsevier Computer Networks, vol. 51, no. 10, pp. 2467-2483, 2007.

[8] M. Myung, "Tutorial on maximum likelihood estimation," Elesevier journal of Mathematical Psychology, no 47, pp. 90-100, 2003.

[9] M. Laaraiedh, S. Avrillon, B. Uguen, "Enhancing Positioning Accuracy through Direct Position Estimators based on Hybrid RSS Data Fusion," in RAS Workshop, IEEE VTC SPRING, 2009.

[10] A. Pal, "Localization Algorithms in Wireless Sensor Networks: Current Approaches and Future Challenges," Network Protocols and Algorithms, Vol. 2, No. 1, pp. 45-74, 2010.

\section{Authors}

Zeinab Abbasi received the B.S. degree in Software Engineering from Shariaty University in 2007. She is a M.S. student in Software Engineering at Payame Noor University (PNU) now. Her research interests are ad hoc network technologies, sensor network technology, image processing and distributed operating systems.

Ahmad Farahi received the $\mathrm{PhD}$ degree in Computer Sciences from Bradford University. He has been working as a full-time faculty member, Assistant Professor and Head of Computer and Information Technology Department in the Payame Noor University. His research interests are programming especially that of educational systems.

Hamid Haj Seyyed Javadi received the B.S., M.S. and PhD degrees in Amir kabir University. He has been working as a full-time faculty member and Assistant Professor of the Shahed University. His research interests are ad-hoc network technology, sensor network technology, distributed operating systems, and heuristic Algorithm. 